\section{Testing Parkinson's metal}

\section{By Lev Osherovich, Senior Writer}

The search for disease-modifying therapeutics in Parkinson's disease has been stymied by a lack of consensus about whether the disease is caused by free radical formation, protein aggregation or mitochondrial dysfunction. Now, a pair of papers in the Proceedings of the National Academy of Sciences makes a case for the first theory by showing the therapeutic effects of blocking iron accumulation in the brain. The studies support the strategy taken by Prana Biotechnology Ltd., which has metal-binding compounds in preclinical development for PD.

PD strikes nearly 1 in 100 people late in life and is characterized by degeneration of the dopaminergic neurons in the substantia nigra, which leads to motor and cognitive defects. The $\$ 2.5$ to $\$ 3$ billion PD market was dominated by GlaxoSmithKline plc's Requip ropinirole, which posted sales of $£ 346$ million ( $\$ 692$ million) last year but went off patent in May. Requip XL, an extended-release formulation codeveloped by GSK and SkyePharma plc, was approved in the U.S. in June and faces competition from Azilect rasagiline, developed by Teva Pharmaceutical Industries Ltd. and H. Lundbeck A/S.

Requip and Azilect work by boosting dopamine activity and thus improving PD symptoms. However, the drugs do little to slow the destruction of neurons.

The two PNAS papers could provide a path for creating disease-modifying drugs. In one article, a team of Chilean and French researchers led by Etienne Hirsch, research director at the Institut National de la Santé et de la Recherche Médicale (INSERM), used mouse and rat models of PD to show that iron plays a critical role in early disease pathogenesis. ${ }^{1}$ Separately, a group of Italian and American researchers led by Luigi Zecca, research director of the Institute for Biomedical Technologies in Milan, reported that a brain pigment mops up PD-associated metals including iron. ${ }^{2}$

\section{Iron on}

Working from past studies suggesting that iron accumulates in the brains of PD patients, ${ }^{3}$ Hirsch's team tested whether a metal-transporting protein called solute carrier family 11 (proton-coupled divalent metal ion transporters), member 2 (SLC11A2; DMT1) was involved in the disease.

The team began by measuring the iron content and DMT1 levels in dopaminergic neurons from PD patients and healthy controls. "We found that DMT1 is present on dopaminergic neurons in the human brain, and its expression is increased in the brains of Parkinsonian patients, who have increased iron content" in the affected tissues, he told SciBX.

The group then moved to a mouse model of PD where a toxic compound called 1-methyl-4-phenyl-1,2,3,6-tetrahydropyridine (MPTP) induces disease by disrupting the normal flow of electrons in mitochondria, a process that goes awry in human PD. The team found that the midbrains of MPTP-treated mice had higher levels of DMT1 and iron than did those of untreated controls.

To determine whether high levels of DMT1 and iron are a cause or a consequence of disease, Hirsch's team looked at the effects of MPTP on mice with partial loss-of-function mutations in DMT1. This partial loss of function decreases the ability of DMT1 to transport iron into cells. Presumably because these mice have less iron in their cells, the DMT1 defect made them more resistant than wild-type mice to the neurotoxic effects of MPTP and another neurotoxic compound called 6-hydroxydopamine.

Hirsch's study suggests that DMT1 imports iron into dopaminergic neurons in response to oxidative stress caused by mitochondrial defects. It thus appears that high iron levels contribute to pathology and are not merely a passive consequence of disease.

Researchers polled by SciBX agreed that lowering DMT1 has diseasemodifying effects in rodents but remained unclear on how iron ultimately contributes to PD.

"There's been a substantial literature showing that iron is a factor in PD," said Ashley Bush, professor of pathology at the Mental Health Research Institute of Victoria and the University of Melbourne. "The novelty here is all about DMT1."

Bush, a cofounder of Prana and a member of the company's R\&D Advisory Board, wanted to know whether iron also enhanced the activity of microglia, a type of innate immune cell that often surrounds PDdamaged neurons. The presumption is that enhanced microglial activity would increase inflammation. Microglia also showed higher DMT1 levels after MPTP treatment, according to Hirsch's study.

Hirsch's paper "proposes a new mechanism of iron accumulation in the substantia nigra of PD patients," said Daniela Berg, professor of neurology at the Hertie Institute for Clinical Brain Research at the University of Tübingen. "However, the reason for elevated iron within the brain needs to be clarified."

She said it's worth looking at whether DMT1 mutations also affect the permeability of the blood-brain barrier (BBB) to iron and other metals.

"The mechanism is uncertain," noted Robert Edwards, professor of neuroscience at the University of California, San Francisco. He added that although the effect of DMT1 on MPTP sensitivity in rodents was interesting and significant, findings in drug-induced rodent models of PD may not translate to humans.

"There have been a lot of things that modulate MPTP toxicity," Edwards cautioned.

One possible mechanism proffered by Hirsch's team proposes that ferrous iron transported into cells by DMT1 stimulates production of free radicals by reacting with hydrogen peroxide, a byproduct of dopamine synthesis (see Figure 1, "Iron in Parkinson's disease"). However, 
Figure 1. Iron in Parkinson's disease. Targeting iron or iron-related reactions in the neuron could lead to potential treatments in Parkinson's disease (PD). Salazar et al. suggest that the increased levels of solute carrier family 11 (proton-coupled divalent metal ion transporters), member 2 (SLC11A2; DMT1) seen in PD patients result in the transport of excess amounts of ferrous iron $\left(\mathrm{Fe}^{2+}\right)$ into dopaminergic neurons (a). ${ }^{1}$ Free $\mathrm{Fe}^{2+}$ engages in a cycle of redox reactions $(\mathbf{b})$ that generate reactive oxygen species such as $\mathrm{HO} \bullet$ that damage the cell via oxidative stress, mitochondrial damage and/or protein aggregation (c). These events lead to neurodegeneration (d). Zecca et al. found that dopaminederived polymers, which form the pigment neuromelanin, can sequester intracellular $\mathrm{Fe}^{2+}(\mathbf{e}) .^{2}$ Such removal of $\mathrm{Fe}^{2+}$ was found to have neuroprotective effects (f).

AMonoamine oxidase $\mathrm{B}$ (MAOB) inhibitors block the formation of $\mathrm{H}_{2} \mathrm{O}_{2}$ and thus antagonize the production of reactive oxygen species. ${ }^{\mathrm{B} M A O B}$ inhibitors also block the degradation of dopamine, allowing for the formation of the $\mathrm{Fe}^{2+}$-sequestering neuromelanin.

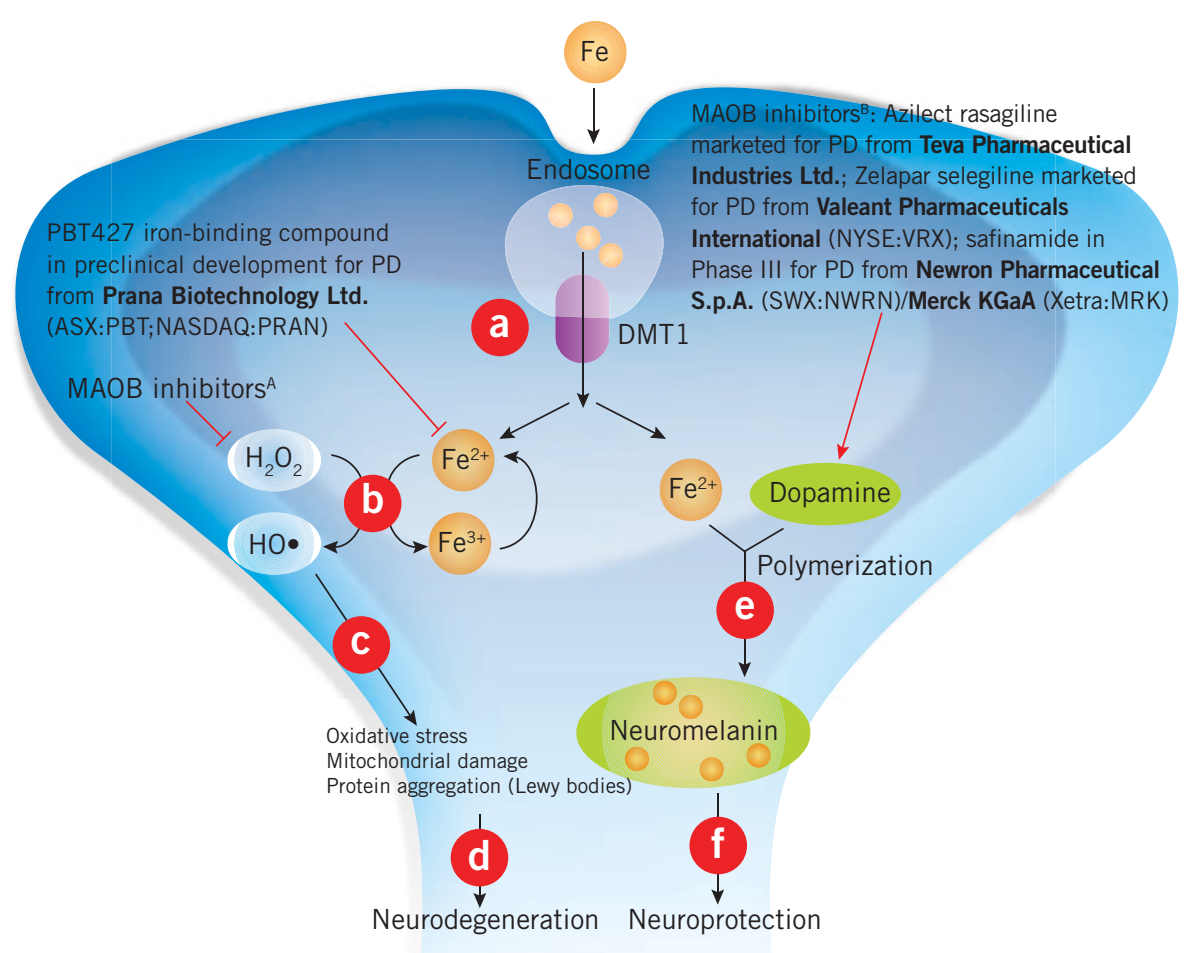

it remains unclear why PD patients and model rodents have higher levels of DMT1 in the first place.

To shore up the hypothesis that intracellular iron exacerbates PD, Edwards suggested testing the effects of blocking other irontransporting proteins such as transferrin in rodent PD models.

Hirsch wants to extend his group's findings into humans by looking at DMT1 activity in patients with hereditary forms of PD. He also wants to identify compounds that modulate DMT1 activity.

The findings reported in the PNAS article have not been patented.

\section{"There's been a substantial literature showing that iron is a factor in PD. The novelty here is all about DMT1." \\ -Ashley Bush, \\ University of Melbourne}

substantia nigra, the putamen, premotor cortex and the cerebellum of elderly patients who were free of PD. Only the first two of these four brain regions are strongly affected in PD. However, neuromelanin-bound iron was present at comparable levels in all four tissues, suggesting that tissues affected by PD are particularly sensitive to iron.

The team also found that, in addition to accumulating iron, the substantia nigra had considerably higher levels of neuromelaninbound lead compared with the other brain tissues. In contrast, other metals accumulated preferentially in other brain regions. These findings were in line with earlier observations that PD risk rises with lead exposure.

Zecca believes that neuromelanin locks away toxic metals into a chemically inert form.

Iron is not the only metal that accumulates in PD-

prone neurons, according to Zecca's paper, also in PNAS. The study surveyed the metal content of neuromelanins, which are dopamine-derived polymeric pigments that give the substantia nigra its dark color.

Zecca's team found that neuromelanins bind and detoxify a range of metals in addition to iron.

"Environmental toxic agents may play a role in neurodegeneration in Parkinson's disease," he said. "The pigments we identified are able to accumulate and immobilize environmental toxic metals like lead, mercury and cadmium in addition to an endogenous metal like iron."

The group analyzed metal-rich neuromelanin granules from the
Indeed, his study suggests that metals complexed to neuromelanin have a lower propensity to oxidize than free metals, and thus do not readily trigger the production of free radicals.

"The ability of pigment to block reactive iron is the last chance for neurons to be protected against iron transport into the cell and iron toxicity," said Zecca.

"This is probably the most comprehensive biochemical analysis of this material to date," said Melbourne's Bush. "Its main message is that these pigments exist throughout the brain."

UCSF's Edwards said the Zecca study was "an important characteriza- 
tion" of an overlooked aspect of PD pathology. "Many people in the field think that neuromelanin is utterly irrelevant, but I'm not one of them," he said.

To firm up the connection between iron levels and PD, Edwards suggested analyzing the neuromelanin content of the dorsal motor nucleus, another highly pigmented brain tissue that is affected by PD. Bush recommended trying to boost neuromelanin levels by increasing the concentration of precursor molecules like cysteine and DOPA, a derivative of dopamine.

Zecca, who also has not patented his findings, said his team is currently studying the membranous envelope surrounding neuromelanins for clues about neurodegeneration.

\section{Therapeutic prospects}

Together, the studies paint a picture in which iron is transported into the cytoplasm by DMT1 and then generates reactive oxygen species that lead to the syndrome of cellular damage seen in PD (see Figure 1, "Iron in Parkinson's disease").

Neuromelanins counteract this effect by sequestering iron and other reactive metals. This pathway provides several potential therapeutic strategies, including blocking iron transport, raising neuromelanin levels, sequestering more iron or lowering the supply of reactants that iron uses to make reactive oxygen species.

Treating the disease could in principle "be as simple as ablating DMT1," said Bush. However, he cautioned that very little is understood about DMT1's natural function in dopaminergic neurons, and that directly targeting DMT1 would be a major challenge because of its location behind the BBB and within the cell.

Indeed, according to Bush, targeting iron is a double-edged sword. Making dopamine requires an enzyme that uses iron, but dopamine synthesis and degradation yields byproducts that can react with iron and other transition metals to make free radicals.

"The brain is using these transition metals for rapid electrochemistry-it has a lot of vulnerabilities," he said. "It's possible that in PD there's a fatigue of antioxidants" needed to keep these metals in check.

On the other hand, the space has seen clinical failures with antioxidants like coenzyme Q10. One ongoing candidate is MitoQ mitoquinone antioxidant, which Antipodean Pharmaceuticals Inc. has in Phase II testing for PD. The company did not return calls seeking comment.

Bush noted that monoamine oxidase $\mathrm{B}(\mathrm{MAOB})$ inhibitors such as Azilect block the degradation of dopamine in a reaction that also yields hydrogen peroxide, a source of reactive oxygen species (see Figure 1, "Iron in Parkinson's disease"). Thus, MAOB inhibitors fight PD on two fronts: raising dopamine levels and choking off free radical formation. Indeed, Azilect may protect neurons independent of its effect on dopamine levels, suggesting that blocking free radical formation could potentially modify the course of PD. ${ }^{4}$

According to Edwards, the mechanisms by which metals and redox chemistry influence PD are "really hard to nail down," making drug development in this area a challenge.

Indeed, few companies are modulating iron toxicity in PD beyond Prana, which is focused on blocking metals to treat neurodegenerative diseases. The company's PBT427, an iron-specific compound, is in preclinical development for PD.

PBT427 is chemically related to Prana's PBT2, an 8-hydroxyquinoquine compound in Phase IIb testing for Alzheimer's disease (AD) and Phase I for Huntington's disease (HD). PBT2 preferentially binds zinc and copper ions, which contribute to toxicity in $\mathrm{AD}$ and $\mathrm{HD}$.

Osherovich, L. SciBX 1(42); doi:10.1038/scibx.2008.1012

Published online Nov. 20, 2008

\section{REFERENCES}

1. Salazar, J. et al. Proc. Natl. Acad. Sci. USA; published online Nov. 14, 2008; doi:10.1073/pnas.0804373105

Contact: Etienne C. Hirsch, Institut National de la Santé et de la Recherche Médicale (INSERM), Paris, France e-mail: hirsch@ccr.jussieu.fr

2. Zecca, L. et al. Proc. Natl. Acad. Sci. USA; published online Nov. 6, 2008; doi:10.1073/pnas.0808768105

Contact: Luigi Zecca, Institute for Biomedical Technologies, Italian National Research Council, Segrate, Italy e-mail: luigi.zecca@itb.cnr.it

Contact: Nicholas Turro, Columbia University, New York, N.Y. e-mail: njt3@columbia.edu

3. Zecca, L. et al. Nat. Rev. Neurosci. 5, 863-873 (2004)

4. Schapira, A.H. et al. Nat. Rev. Drug Discov. 5, 845-854 (2006)

\section{COMPANIES AND INSTITUTIONS MENTIONED}

Antipodean Pharmaceuticals Inc., San Francisco, Calif. GlaxoSmithKline plc (LSE:GSK; NYSE:GSK), London, U.K. H. Lundbeck A/S (CSE:LUN), Copenhagen, Denmark Hertie Institute for Clinical Brain Research, Tübingen, Germany Institut National de la Santé et de la Recherche Médicale, Paris, France

Institute for Biomedical Technologies, Milan, Italy Mental Health Research Institute of Victoria, Victoria, Australia Prana Biotechnology Ltd. (ASX:PBT; NASDAQ:PRAN), Melbourne, Australia

SkyePharma plc (LSE:SKP), London, U.K. Teva Pharmaceutical Industries Ltd. (NASDAQ:TEVA), Jerusalem, Israel

University of California, San Francisco, Calif.

University of Melbourne, Victoria, Australia

University of Tübingen, Tübingen, Germany 